PROCEEDINGS OF THE

AMERICAN MATHEMATICAL SOCIETY

Volume 129, Number 3, Pages 663-666

S 0002-9939(00)05627-6

Article electronically published on August 30, 2000

\title{
CHARACTERIZATION OF DISCRIMINATOR VARIETIES
}

\author{
DIEGO VAGGIONE \\ (Communicated by Carl G. Jockusch, Jr.)
}

\begin{abstract}
We prove that a variety $\mathcal{V}$ is a discriminator variety if and only if $\mathcal{V}$ has the Fraser-Horn property and every member of $\mathcal{V}$ is representable as a Boolean product whose factors are directly indecomposable or trivial.
\end{abstract}

A variety has the Fraser-Horn property [3] if every congruence on a product $A_{1} \times A_{2}$ is of the form $\theta_{1} \times \theta_{2}$. Examples of varieties with this property are congruence distributive varieties and congruence modular varieties in which no non-trivial member has a trivial subalgebra [8]. In this paper we prove the following:

Theorem. For a variety $\mathcal{V}$ the following are equivalent:

(a) $\mathcal{V}$ is a discriminator variety.

(b) $\mathcal{V}$ has the Fraser-Horn property and every member of $\mathcal{V}$ is representable as a Boolean product whose factors are directly indecomposable or trivial.

We remit to [2] for notation and basic facts on Boolean products and discriminator varieties. The concept of decomposition operation plays a deep role in the proof. A decomposition operation on an algebra $A$ is a homomorphism $d: A \times A \rightarrow A$ satisfying:

$d(x, x) \approx x$ $d(d(x, y), z) \approx d(x, z) \approx d(x, d(y, z))$.

Given a pair $\theta, \delta$ of complementary factor congruences we have associated a decomposition operation defined by $d_{\theta, \delta}(a, b)=$ the unique $c \in A$ such that $(c, a) \in \theta$ and $(c, b) \in \delta$. Reciprocally, given a decomposition operation $d$, the relations $\theta_{d}=$ $\{(x, y): d(x, y)=y\}$ and $\delta_{d}=\{(x, y): d(x, y)=x\}$ are a pair of complementary factor congruences. These maps are mutually inverse [4].

For an algebra $A$ let $\Delta$ denote the diagonal congruence on $A$.

Proof of the Theorem. (a) $\Rightarrow$ (b) Since $\mathcal{V}$ is congruence distributive it has the FraserHorn property [1. The Boolean representation is the well known result of BulmanFleming, Keimel and Werner [2].

(b) $\Rightarrow($ a) Since $\mathcal{V}$ has the Fraser-Horn property, we have that:

(1) For every $A \in \mathcal{V}$, the set $F C(A)$ of all factor congruences forms a Boolean sublattice of the congruence lattice of $A$.

(2) If $\varphi: A \rightarrow B$ is an onto homomorphism and $d$ is a decomposition operation on $A$, then the equation $\varphi(d)(\varphi(a), \varphi(b))=\varphi(d(a, b))$ defines a decomposition operation $\varphi(d)$ on $B$.

Received by the editors March 2, 1999 and, in revised form, May 14, 1999.

2000 Mathematics Subject Classification. Primary 08A05, 08A30, 08A40, 08B10, 06E15.

The author's research was supported by CONICOR and SECYT (UNC). 
(1) is proved in 11, 1.4]. To prove (2) suppose (w.l.o.g.) that $A=A_{1} \times A_{2}$ and $d\left(\left(a_{1}, a_{2}\right),\left(b_{1}, b_{2}\right)\right)=\left(a_{1}, b_{2}\right)$, and use the fact that $\operatorname{ker} \varphi$ factorizes 1

Next let $A \subseteq \Pi\left\{A_{i}: i \in I\right\}$ be a Boolean product. For $i \in I$, let $\theta_{i}=\{(x, y) \in$ $\left.A^{2}: x(i)=y(i)\right\}$. Suppose that there is a set $J \subseteq I$ such that every $A_{i}$, with $i \in J$, is directly indecomposable and $\bigcap\left\{\theta_{i}: i \in J\right\}=\Delta$. We will prove that

(3) $\left\{\theta_{i}: i \in I\right\}-\{\Delta\}=\{\cup \mathbf{m}: \mathbf{m}$ is a maximal ideal of $F C(A)\}-\{\Delta\}$.

For a set $B$ define $\eta^{B}(a, b, c, d)=c$, if $a=b$ and $\eta^{B}(a, b, c, d)=d$, if $a \neq b$. By [6, 2.3] there is a 4 -ary operation $h$ on $A$ such that the algebra $\langle A, h\rangle$ generates a discriminator variety and

(4) $\langle A, h\rangle \subseteq \Pi\left\{\left\langle A_{i}, \eta^{A_{i}}\right\rangle: i \in I\right\}$ is a Boolean product with simple or trivial factors.

Let $\theta, \theta^{*}$ be a pair of complementary factor congruences of $A$. Since $\theta_{i}=\left(\theta_{i} \vee\right.$ $\theta) \cap\left(\theta_{i} \vee \theta^{*}\right) 3$ and each $\theta_{i}$, with $i \in J$, is indecomposable, we have that $\theta \subseteq \theta_{i}$ or $\theta^{*} \subseteq \theta_{i}$, for every $i \in J$. Since $\bigcap\left\{\theta_{i}: i \in J\right\}=\Delta$, we obtain that $\bigcap\left\{\theta_{i}: \theta \subseteq \theta_{i}\right\}$ and $\bigcap\left\{\theta_{i}: \theta^{*} \subseteq \theta_{i}\right\}$ are a pair of complementary factor congruences of $A$, which by (1) implies that $\theta=\bigcap\left\{\theta_{i}: \theta \subseteq \theta_{i}\right\}$. Thus $\theta$ is a congruence on $\langle A, h\rangle$ (note that the $\theta_{i}$ are congruences of $\left.\langle A, h\rangle\right)$ and we have proved that

(5) $F C(A)=F C(\langle A, h\rangle)$.

Since principal congruences in discriminator varieties are factor congruences [10] (6) of 2.2], (5) implies that

(6) For $(x, y) \in A^{2}$ there is a least factor congruence containing the pair $(x, y)$. Since $\langle A, h\rangle$ generates a discriminator variety, we have that

(7) The natural map

$$
\langle A, h\rangle \rightarrow \Pi\{\langle A, h\rangle / \cup \mathbf{m}: \mathbf{m} \text { is a maximal ideal of } F C(A)\}
$$

is a Boolean representation with simple or trivial factors.

Thus (3) follows from (4), (7) and the uniqueness of Boolean representations in discriminator varieties $[5,8.3]$. We note that (3) and (b) imply that

(8) For every $A \in \mathcal{V}, A / \cup \mathbf{m}$ is directly indecomposable or trivial, for every maximal ideal of $F C(A)$.

Next we will prove 2 that

(9) If $A$ is directly indecomposable and $B \subseteq A$, then $B$ is directly indecomposable or trivial.

Suppose to the contrary that $B$ is decomposable. Let $x_{0}$ be a point in the Cantor Discontinuum $C$. Give $A$ the discrete topology. Let $D=\left\{f \in A^{C}: f\right.$ is continuous and $\left.f\left(x_{0}\right) \in B\right\}$. Note that $D \subseteq \Pi\left\{D_{x}: x \in C\right\}$ is a Boolean product where $D_{x}=A$ if $x \neq x_{0}$ and $D_{x_{0}}=B$. Further note that $\bigcap_{x \in C-\left\{x_{0}\right\}} \theta_{x}=\Delta\left(C-\left\{x_{0}\right\}\right.$ is dense). Thus (3) and (8) produce a contradiction since $D / \theta_{x_{0}} \cong B$.

Let $c_{1}, c_{2}$ be new constant symbols and let $d_{1}, d_{2}, \ldots$ be new binary function symbols. Let $\mathcal{V}_{e}$ be the variety axiomatized by a set of identities defining $\mathcal{V}$ plus the following identities:

$$
\begin{aligned}
& d_{i}(x, x) \approx x, \\
& d_{i}\left(d_{i}(x, y), z\right) \approx d_{i}(x, z) \approx d_{i}\left(x, d_{i}(y, z)\right) \\
& d_{i}(f(\vec{x}), f(\vec{y})) \approx f\left(d_{i}\left(x_{1}, y_{1}\right), \ldots, d_{i}\left(x_{n}, y_{n}\right)\right), \\
& d_{i}\left(c_{1}, c_{2}\right) \approx c_{2}, f \text { an } n \text {-ary function symbol of } \mathcal{V}, n \geq 1, i=1,2, \ldots
\end{aligned}
$$

Note that $\left\langle A, a, b, d_{1}, \ldots\right\rangle \in \mathcal{V}_{e}$ iff $A \in \mathcal{V}$ and each $d_{i}$ is a decomposition operation on $A$ such that $(a, b) \in \theta_{d_{i}}$. Let $F$ be the free $\mathcal{V}_{e^{-a l g e b r a}}$ freely generated by $z$ and

\footnotetext{
${ }^{1}$ Indeed condition (2) is equivalent to the Fraser-Horn property.

${ }^{2}$ The argument we will use was extracted from [1].
} 
let $F_{r}$ be the reduct of $F$ to the language of $\mathcal{V}$. Since $F_{r} \in \mathcal{V}$, by (6) there exists a factor congruence $\theta$, which is the least factor congruence on $F_{r}$ containing the pair $\left(c_{1}, c_{2}\right)$. Let $\delta$ be the complement of $\theta$ in $F C\left(F_{r}\right)$. Note that

(10) $d_{\theta, \delta}\left(c_{1}, c_{2}\right)=c_{2}$.

Since each $d_{i}^{F}$ is a decomposition operation on $F_{r}$ satisfying $\left(c_{1}, c_{2}\right) \in \theta_{d_{i}^{F}}$, we have

(11) $\forall a, b \in F_{r} \quad d_{\theta, \delta}(a, b)=b \rightarrow d_{i}^{F}(a, b)=b$.

For each term $t(z)$ in the language of $\mathcal{V}_{e}$, define a term $t(z)^{*}$ with variables in $\{x, y, z\}$ as follows:

$c_{1}^{*}=x$,

$c_{2}^{*}=y$

$s^{*}=s$, for $s \in\{z\} \cup\{$ constant symbols of $\mathcal{V}\}$,

$f\left(t_{1}, \ldots, t_{n}\right)^{*}=f\left(t_{1}^{*}, \ldots, t_{n}^{*}\right), f$ an $n$-ary function symbol of $\mathcal{V}$,

$d_{i}\left(t_{1}, t_{2}\right)^{*}=t_{2}^{*}, i=1,2, \ldots$

We will follow the usual custom of thinking of $d_{\theta, \delta}\left(z, c_{1}\right) \in F$ as a term $t(z)$, which we prefer to write as $t\left(c_{1}, c_{2}, z\right)$ to refer to all the occurrences of $c_{1}$ and $c_{2}$ in $t(z)$. The goal will be to show that $d_{\theta, \delta}\left(z, c_{1}\right)^{*}=t\left(c_{1}, c_{2}, z\right)^{*}$ provides a discriminator term for $\mathcal{V}$, i.e. we will prove that for every directly indecomposable $A \in \mathcal{V}$ and every $a, b, c \in A$, we have that $t\left(c_{1}, c_{2}, z\right)^{*}(a, b, c)=c$, if $a=b$ and $t\left(c_{1}, c_{2}, z\right)^{*}(a, b, c)=a$, otherwise. Note that by (9) we can suppose that $A$ is generated by $\{a, b, c\}$. Let $\pi_{1}, \pi_{2}: A \times A \rightarrow A$ be the canonical projections. Let $k$ be such that each $d_{i}$, with $i>k$, does not occur in $t\left(c_{1}, c_{2}, z\right)$. First suppose that $a=b$. Since $\langle A, a, b, \overbrace{\pi_{2}, \ldots, \pi_{2}}^{k}, \pi_{1}, \pi_{1}, \ldots\rangle \in \mathcal{V}_{e}$, there is a morphism

$$
\begin{aligned}
\varphi: F & \rightarrow\left\langle A, a, b, \pi_{2}, \ldots, \pi_{2}, \pi_{1}, \pi_{1}, \ldots\right\rangle \\
z & \rightarrow c .
\end{aligned}
$$

Since $d_{\theta, \delta}\left(z, d_{\theta, \delta}\left(z, c_{1}\right)\right)=d_{\theta, \delta}\left(z, c_{1}\right)$, by (11) we have that $d_{i}^{F}\left(z, d_{\theta, \delta}\left(z, c_{1}\right)\right)=$ $d_{\theta, \delta}\left(z, c_{1}\right), i=1,2, \ldots$. Further note that

$$
\varphi\left(d_{\theta, \delta}\left(z, c_{1}\right)\right)=\varphi\left(t\left(c_{1}, c_{2}, z\right)\right)=t\left(c_{1}, c_{2}, z\right)^{*}(a, b, c) .
$$

Thus we have

$$
t\left(c_{1}, c_{2}, z\right)^{*}(a, b, c)=\varphi\left(d_{k+1}\left(z, d_{\theta, \delta}\left(z, c_{1}\right)\right)\right)=\pi_{1}\left(c, \varphi\left(d_{\theta, \delta}\left(z, c_{1}\right)\right)\right)=c .
$$

Finally suppose that $a \neq b$. Let

$$
\begin{aligned}
\gamma: F & \rightarrow\left\langle A, a, b, \pi_{2}, \ldots, \pi_{2}, \pi_{2}, \pi_{2}, \ldots\right\rangle \\
z & \rightarrow c .
\end{aligned}
$$

Note that by (2) we have a decomposition operation $\gamma\left(d_{\theta, \delta}\right)$. Since $\left(c_{1}, c_{2}\right) \in \theta=$ $\theta_{d_{\theta, \delta}}$, we have that $(a, b) \in \theta_{\gamma\left(d_{\theta, \delta}\right)}$, which implies that $\theta_{\gamma\left(d_{\theta, \delta}\right)}=A \times A$, because $A$ is directly indecomposable. Thus $\gamma\left(d_{\theta, \delta}\right)=\pi_{2}$ and hence

$$
t\left(c_{1}, c_{2}, z\right)^{*}(a, b, c)=\gamma\left(d_{\theta, \delta}\left(z, c_{1}\right)\right)=\gamma\left(d_{\theta, \delta}\right)\left(\gamma(z), \gamma\left(c_{1}\right)\right)=\pi_{2}(c, a)=a .
$$

$(\mathrm{b}) \Rightarrow(\mathrm{a})$ was proved in [7] with the restriction that the Boolean representations have no trivial factors. That proof is based on the concept of central element [9] which cannot be used in general since central elements exist only in varieties in which no non-trivial algebra has a trivial subalgebra. We conclude the paper with the following question: 
Is it possible to replace in the above theorem the Fraser-Horn property by the property that for every $A \in \mathcal{V}$, the set $F C(A)$ forms a Boolean sublattice of the congruence lattice of $A$ ?

\section{REFERENCES}

[1] D. BIGELOW and S. BURRIS, Boolean algebras of factor congruences, Acta Sci. Math. $\mathbf{5 4}$ (1990), 11-20. MR 91j:08012

[2] S. BURRIS and H. SANKAPPANAVAR, A course in Universal Algebra, Springer-Verlag, New York, 1981. MR 83k:08001

[3] G. A. FRASER and A. HORN, Congruence relations in direct products, Proc. Amer. Math. Soc. 26 (1970), 390-394. MR 42:169

[4] R. McKenZIE, G. McNULTY and W. TAYLOR, Algebras, Lattices, Varieties, Volume 1, The Wadswort \& Brooks/Cole Math. Series, Monterey, California (1987). MR 88e:08001

[5] D. VAGGIONE, Locally Boolean spectra, Algebra Universalis 33 (1995), 319-354. MR 95m:08007

[6] D. VAGGIONE, Free algebras in discriminator varieties, Algebra Universalis 34 (1995), 391403. MR 97a:08019

[7] D. VAGGIONE, $\mathcal{V}$ with factorable congruences and $\mathcal{V}=I \Gamma^{a}\left(\mathcal{V}_{D I}\right)$ imply $\mathcal{V}$ is a discriminator variety, Acta Sci. Math. 62 (1996), 359-368. MR 97h:08012

[8] D. VAGGiOne, Modular varieties with the Fraser-Horn property, Proc. Amer. Math. Soc. 127 (1999), 701-708. CMP 99:06

[9] D. VAGGIONE, Central elements in varieties with the Fraser-Horn property, Advances in Math. 148 (1999), 193-202.

[10] H. WERNER, Discriminator algebras, algebraic representation and model theoretic properties, Akademie-Verlag, Berlin, 1978. MR 80f:08009

Facultad de Matemática, Astronomía y Física (Famaf), Universidad Nacional de Córdoba - Ciudad Universitaria Córdoba 5000, Argentina

E-mail address: vaggione@mate.uncor.edu 